\title{
Identification and characterization of cysteinyl exposure in proteins by selective mercury labeling and nano-electrospray ionization quadrupole time-of-flight mass spectrometry
}

\author{
Meiling Lu ${ }^{1,2 *}$, Xing-Fang $\mathrm{Li}^{3}, \mathrm{X}$. Chris $\mathrm{Le}^{3}$, Michael Weinfeld ${ }^{2}$ and Hailin Wang ${ }^{1 * \star}$ \\ ${ }^{1}$ State Key Laboratory for Environmental Chemistry and Ecotoxicology, Research Centre for Eco-Environmental Sciences, Chinese Academy of \\ Sciences, Shuangqing Road 18, Haidian District, Beijing 100085, P.R. China \\ ${ }^{2}$ Department of Oncology, Cross Cancer Institute and University of Alberta, 11560 University Avenue, Edmonton, T6G 1Z2, Canada \\ ${ }^{3}$ Department of Laboratory Medicine and Pathology, Faculty of Medicine and Dentistry, University of Alberta, Edmonton, T6G 2G3, Canada
}

Received 26 January 2010; Revised 8 March 2010; Accepted 15 March 2010

We describe a method for probing surface-exposed cysteines in proteins by selective labeling with $p$ hydroxymercuribenzoate (PMB) combined with nano-electrospray ionization mass spectrometric analysis (nanoESI-MS). The rapid, stoichiometric, and specific labeling by PMB of surface-exposed cysteines allows for characterization of the accessibility of the cysteines using a single MS analysis. Moreover, by taking advantage of the large mass shift of $321 \mathrm{Da}$, unique isotopic pattern, and enhanced MS signal of PMB-labeled cysteine-containing peptide fragments, the surface-exposed cysteines in proteins can be accurately identified by peptide mapping. The number and sites of reactive cysteines on the surface of human and rat hemoglobins ( $\mathrm{hHb}$ and $\mathrm{rHb}$ ) were identified as examples. Collision-induced dissociation tandem mass spectrometric (MS/MS) analysis of specific peptides further confirmed the selective labeling of PMB in $\mathrm{hHb}$. The subtle difference between the different cysteine residues in $\mathrm{rHb}$ was also evaluated by multiple PMB titrations. The difference between the two cysteines in their environment may partially explain their reaction specificity. Cysteine 125 in the $\beta$ unit of $\mathrm{rHb}$ is exposed on the surface, explaining its reactivity with glutathione. Cysteine 13 in the $\alpha$ subunit of $\mathrm{rHb}$ is much less exposed, and is located in a hydrophobic pocket, a conclusion that is consistent with the previous observation of its selective binding with dimethylarsinous acid, a reactive arsenic metabolite. The method is potentially useful for probing cysteines in other biologically important proteins and for studying proteins that are associated with conformational or structural changes induced by denaturing processes, protein modifications, proteinprotein interactions and protein assemblies. Copyright (C) 2010 John Wiley \& Sons, Ltd.

Cysteine is a moderately hydrophobic amino acid that may be exposed on a protein or be shielded by tertiary or quaternary protein structures. It can be involved in protein folding by forming disulfide bonds, maintenance of structural integrity and stability, ${ }^{1-3}$ formation of functional domains (e.g. catalysis, DNA binding), ${ }^{4-6}$ metal chelation, mediation of redox state (e.g. S-conjugation, glutathionylation, S-nitrosylation), and regulation of signal transduction (S-nitrosylation). ${ }^{7-11}$ The accessibility of cysteine is an important means for proteins to mediate their conformations and functions. ${ }^{1,12-15}$ Several studies have demonstrated that cysteines on the protein surface that are solvent-accessible are generally more reactive and biochemically important. ${ }^{13,14,16}$

*Correspondence to: M. Lu, Department of Oncology, Cross Cancer Institute and University of Alberta, 11560 University Avenue, Edmonton, T6G 1Z2, Canada.

E-mail: meiling@ualberta.net

${ }^{* *}$ Correspondence to: H. Wang, State Key Laboratory for Environmental Chemistry and Ecotoxicology, Research Centre for EcoEnvironmental Sciences, Chinese Academy of Sciences, Shuangqing Road 18, Haidian District, Beijing 100085, P.R. China.

E-mail: hlwang@rcees.ac.cn
For example, glutathionylation at exposed Cys124 and Cys141 of p53 caused by oxidative stress can inhibit the binding of p53 to DNA. ${ }^{13}$ The exposure of the buried cysteine in the $\alpha$ unit of human hemoglobin that is induced by the mutation of tyrosine at $\beta 35$ to phenylalanine may destabilize the hemoglobin tetramer and lead to hemolysis. ${ }^{16}$ The access to surface cysteines has been exploited as an indicator for studying protein assembly and denaturation, and forceinduced conformational changes. ${ }^{17-19}$ Understanding the biological functions and associated conformational changes of thiol-containing proteins also benefits characterization of the spatial environment around the cysteines. Traditionally, whether cysteines are exposed on the protein surface or buried inside the protein is measured by spectrometric titration, ${ }^{16,20}$ fluorescence labeling, ${ }^{21}$ and labor-intensive point mutation of cysteines. ${ }^{22}$

A range of cysteine-specific reagents have been applied to probe the cysteinyl reactivity in various proteins. ${ }^{23-28} p$ Hydroxymercuribenzoate (PMB) is one of such reagents. It only labels free cysteines specifically due to the high affinity 
of monovalent mercury for the nucleophilic sulfhydryl group. ${ }^{29}$ Moreover, due to its bulky size and hydrophilic nature (negative charge), PMB is difficult to diffuse into the hydrophobic core of proteins, and only labels free cysteines on the protein surface. PMB labeling has been applied to identify the exposed cysteines in proteins by spectrometric titration without denaturation of proteins. ${ }^{16,20}$ It was also proposed to label denatured proteins for measuring the number of total cysteines on proteins. $^{30}$ More recently, PMB-based beads were applied to enrich cysteine-containing peptides as a complementary method for improving the sequence coverage and enhancing the identification confidence of proteins. ${ }^{31}$ However, no specific method based on PMB labeling and MS detection has been exploited for studying the cysteine environment in proteins.

Here we combine selective mercury labeling with mass spectrometry technology for fast identification and characterization of surface-exposed cysteines in proteins. To demonstrate the application of this method, human and rat hemoglobins were chosen as model proteins. The number and sites of cysteines in hemoglobins vary greatly from one species to another, which could lead to different tolerance to exogenous stresses. With the method developed here, the number and location of the exposed cysteines in both hemoglobin of human and rat were identified.

\section{EXPERIMENTAL}

Caution: $p$-Hydroxymercuribenzoate (PMB) is toxic, and special care should be taken.

\section{Reagents}

PMB, trypsin (TPCK treated, proteomics grade), and standard hemoglobins of human and rat $\mathrm{hHb}$ and $\mathrm{rHb}^{\mathrm{WT}}$ ) were purchased from Sigma (St. Louis, $\mathrm{MO}, \mathrm{USA})$. Another rat hemoglobin $\left(\mathrm{rHb}^{\mathrm{F} 344}\right)$ was extracted from blood of an F344 rat ( 16 weeks old) following a published protocol. ${ }^{32}$ Both methanol and water were of HPLC grade, and purchased from Fisher Scientific (Fair Lawn, NJ, USA). All other reagents used were of analytical grade.

\section{Single and multiple titration of hemoglobin using PMB}

$\mathrm{Hb}$ ( $5 \mathrm{nmol} ; 50 \mu \mathrm{M}$ in $100 \mu \mathrm{L}$ sodium phosphate buffer (50 mM, pH 7.0)) was reacted with $0,1,2,4,6,12$, or 24 -fold molar excess of PMB by addition of stock PMB (50 mM PMB in $50 \mathrm{mM}$ sodium phosphate buffer, $\mathrm{pH}$ 7.0) at room temperature for $10 \mathrm{~min}$. The resultant mixtures were subjected to desalting using a BioSpin column (Bio-Rad Laboratories, Hercules, CA, USA) for removal of unreacted PMB. The filtrates were then diluted to a final concentration of $\mathrm{Hb}$ of $\sim 2 \mu \mathrm{M}$ using $10 \%$ methanol/water solution containing $0.001 \%$ formic acid, and subjected to nanoelectrospray ionization mass spectrometric (nanoESI-MS) analysis.

\section{Trypsin digestion of hemoglobin}

The reaction mixtures after incubation as prepared above were first centrifuged using Microcon-10 concentrators (molecular weight (MW) cutoff: $10 \mathrm{kDa}$; Millipore, Milford, MA, USA) against the phosphate buffer ( $50 \mathrm{mM}, \mathrm{pH} 7.0)$ to remove unreacted $\mathrm{PMB}$ by centrifugation at $13000 \mathrm{rpm}$ and $4^{\circ} \mathrm{C}$. The resultant PMB-labeled $\mathrm{Hb}$ was then exchanged into digestion buffer $(50 \mathrm{mM}$ ammonia bicarbonate, $\mathrm{pH} 7.8)$, followed by digestion using trypsin with a mass ratio of protein to enzyme of 30:1 for $18 \mathrm{~h}$. The resultant peptide mixtures were subjected to Ziptip C18 (Millipore, Milford, MA, USA) purification. The eluent from the Ziptip C18 was directly loaded onto a nanoESI tip and subjected to nanoESIMS analysis.

\section{NanoESI-MS analysis of PMB-Hb reaction products}

We used a previously described system with slight modification. $^{32,33}$ Briefly, a nanoESI source (Protana, Denmark) was coupled to a QSTAR Pulsar I mass spectrometer (Applied Biosystem/MDS Sciex, Concord, Ontario, Canada). The conductive capillary tip for nanoESI (Proxeon Biosystem, Denmark) filled with samples was positioned onto the nanoESI source using a three-dimensional positioner. The system was operated in the positive ionization mode with an ionization spray voltage of $950 \mathrm{~V}$, first declustering potential (DP1) of $65 \mathrm{~V}$, second declustering potential (DP2) of $10 \mathrm{~V}$, and focusing potential (FP) of $215 \mathrm{~V}$. The instrument was routinely calibrated using standard horse skeletal apomyoglobin (Sigma-Aldrich, St. Louis, MO, USA) as an external standard. Each spectrum ( $m / z$ 600-1500) was collected for $2 \mathrm{~min}$ (120 cycles). The resulting spectra were displayed and deconvoluted using Analyst/Bioanalyst software (Applied Biosystem/MDS Sciex), and further processed using Igor Pro Software (WaveMetrics, Lake Oswego, OR, USA)

For MS/MS analysis of peptides of interest, the second quadrupole of the QSTAR system was used to select the parent ions which were fragmented into a series of product ions in the third quadrupole by applying a collision energy of $22 \mathrm{~V}$, and a collision gas of 6 (absolute value). The product ions were then transferred to the time-of-flight tube and detected by a multichannel plate detector.

\section{RESULTS AND DISCUSSION}

\section{Measurement of the number of surface-exposed cysteines in $\mathrm{hHb}$}

Hemoglobin is a tetramer protein consisting of two $\alpha$ units and two $\beta$ units. A heme group is attached in each unit through non-covalent interaction. The nanoESI-MS analysis of untreated human hemoglobin ( $\mathrm{hHb}, 2 \mu \mathrm{M})$ showed two major peaks of intact $\alpha$ (peak 3, $15742 \mathrm{Da})$ and intact $\beta$ units (peak 7, $16482 \mathrm{Da}$ ). Both intact $\alpha$ and $\beta$ units contained one heme group (bottom trace, Fig. 1(A)). In addition, two minor peaks of apo $\alpha$ (peak 1, $15126 \mathrm{Da}$ ) and apo $\beta$ units (peak 4, $15866 \mathrm{Da})$ were observed, resulting from a loss of one heme group (616 Da) from the corresponding unit during nanoESI. The measured molecular weights of these units are consistent with our previous work ${ }^{32,33}$ and that reported in the SwissProt database. ${ }^{34}$ 

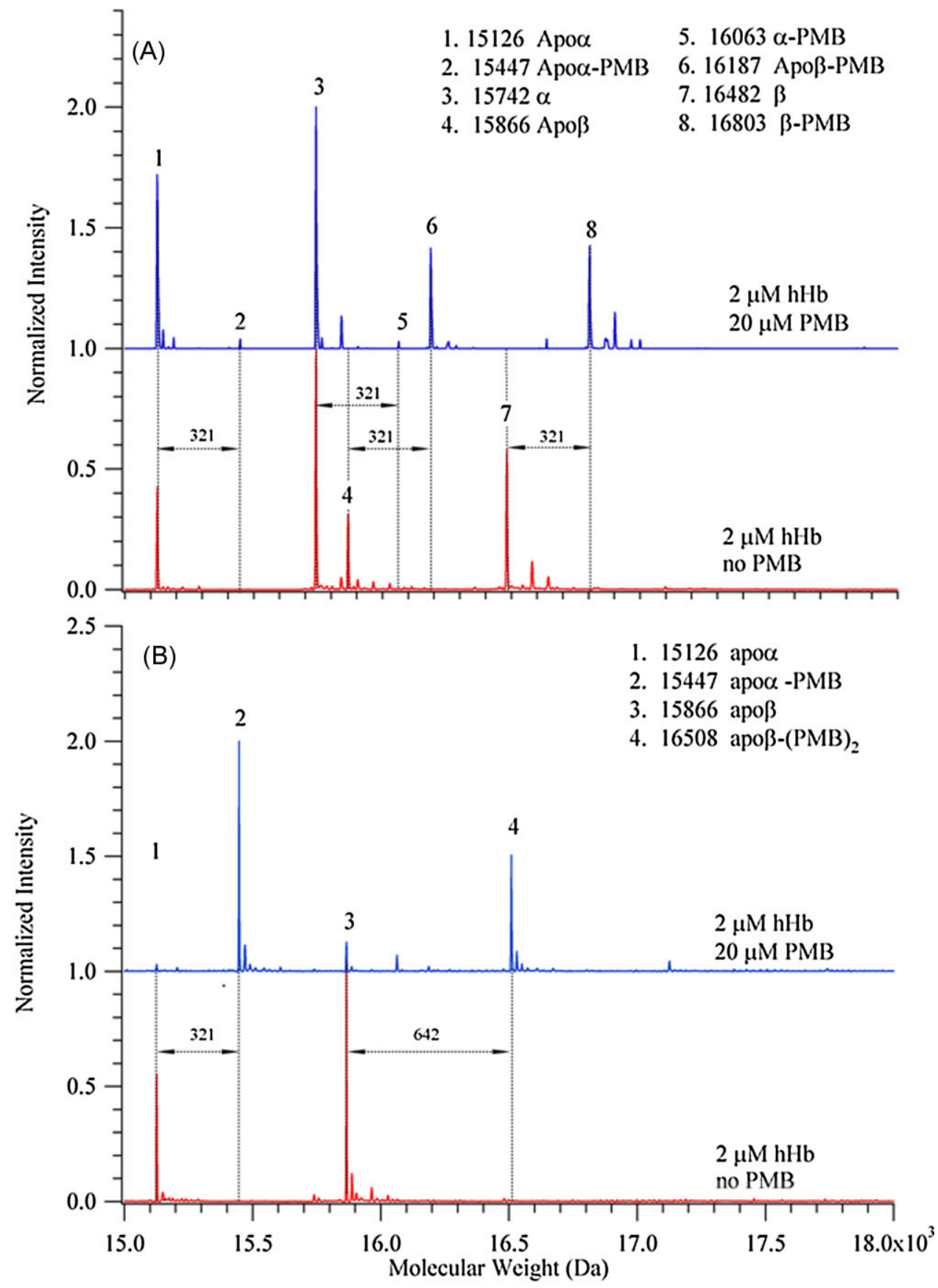

Figure 1. Comparison of the mass spectra of hemoglobin labeled by PMB under native (A) and denatured (B) conditions. (A). Mass spectra of the native $\mathrm{hHb}$ and PMB-labeled native $\mathrm{hHb}$ showing that $\mathrm{PMB}$ can react with the $\beta$ unit of $\mathrm{hHb}$ predominantly and form a 1:1 complex ( $\beta$ unit to PMB), suggesting the presence of two exposed cysteines for one $\mathrm{hHb}$ tetramer. (B) Mass spectra of the denatured $\mathrm{hHb}$ and PMB-labeled denatured $\mathrm{hHb}$ showing that PMB can react with both the $\alpha$ and $\beta$ units of $\mathrm{hHb}$ and form 1:1 and 1:2 complexes, respectively ( $\mathrm{hHb}$ unit to $\mathrm{PMB}$ ). The peaks identified are listed in the figure with their measured molecular weights. The detailed experimental conditions are described in the Experimental section.

To measure the number of surface-exposed cysteines in native $\mathrm{hHb}, \mathrm{hHb}(2 \mu \mathrm{M})$ was reacted with excess PMB $(20 \mu \mathrm{M})$ in phosphate buffer $(50 \mathrm{mM}, \mathrm{pH} 7.0)$ without any denaturants. The nanoESI-MS analysis of excess PMB titrated $\mathrm{hHb}$ showed the disappearance of the apo $\beta$ unit and the intact $\beta$ unit, accompanied by the appearance of two complexes, apo $\beta$-PMB (peak 6, $16187 \mathrm{Da}$ ) and $\beta$-PMB (peak 8, $16803 \mathrm{Da}$ ) (top trace in Fig. 1(A)). Both complexes have a molecular mass 321 Da higher than the corresponding apo $\beta$ unit (peak 4, $15866 \mathrm{Da}$ ) and intact $\beta$ unit (peak 7, $16482 \mathrm{Da}$ ). The increase in mass by $321 \mathrm{Da}$ is consistent with the binding of one PMB with one cysteine in the $\beta$ unit, indicating the formation of a 1:1 complex as shown in the reaction scheme (Scheme 1). Although there are two free cysteines ( $\beta 93$ and $\beta 112$ ) in the $\beta$ unit, no peak corresponding to a complex of other stoichiometry was observed. These results suggest that 


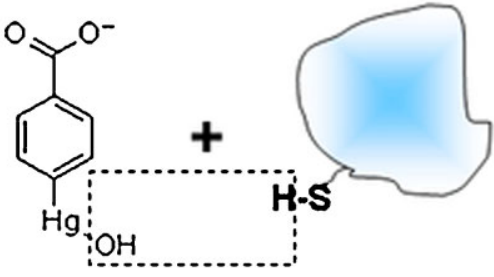

PMB<smiles>O=C([O-])c1ccc(O)cc1</smiles>

PMB
Native protein with exposed cysteine

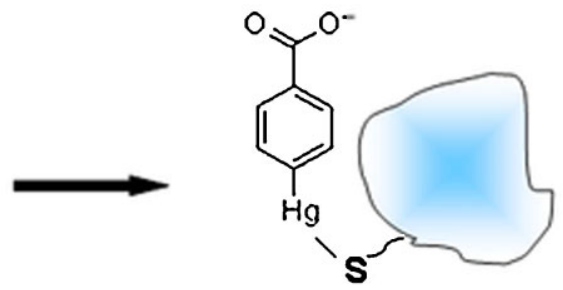

Native protein labeled with $\mathrm{PMB}$

$321 \mathrm{Da}$ increase in MW

\section{No Reaction}

Scheme 1. PMB binding to cysteine residues exposed on the surface of native proteins induces molecular weight increase of the protein by $321 \mathrm{Da}$.

only one cysteine in the $\beta$ unit is highly exposed or solventaccessible. This cysteine can be easily targeted by the selective labeling of bulky and hydrophilic PMB molecules.

Although excess PMB was used for labeling, more than 95\% of apo $\alpha$ (peak 1, 15126 Da) and the $\alpha$ unit (peak 3, 15742 Da) could not be labeled by PMB, judging from the peak heights of the apo $\alpha$-PMB complex (peak 2, $15447 \mathrm{Da}$ ) and the $\alpha$-PMB complex (peak 5, 16063 Da) (Fig. 1(A)) that represent less than $5 \%$ of the unreacted $\alpha$ unit. Both the complexes have a binding stoichiometry of 1:1 (one PMB molecule bound to each $\alpha$ unit). The results indicate that the single cysteine ( $\alpha 104)$ in the $\alpha$ unit is buried and not accessible by PMB. This observation is consistent with the quaternary structure of hemoglobin in which $\alpha 104$ sits at the interface between the $\alpha$ and $\beta$ units (PDB database). With the preservation of the quaternary structure of human hemoglobin, $\alpha 104$ is poorly exposed to solvent.

In contrast, when the same amount of PMB was reacted with $\mathrm{hHb}$ under denaturing conditions (e.g. in the presence of $4 \mathrm{M}$ urea), it was found that each $\alpha$ unit can bind to one PMB molecule (peak 2), and each $\beta$ unit can bind to two PMB molecules (peak 4, Fig. 1(B)). Therefore, one hHb molecule which contains two $\alpha$ and two $\beta$ units can bind six PMB molecules in total under denaturing conditions. The number of $\mathrm{PMB}$ molecules bound to $\mathrm{hHb}$ is consistent with the available cysteines in each unit, indicating that excess PMB can label all the free cysteines when they are all exposed through denaturation of the protein.

Although the $\mathrm{hHb}$ tetramer has a total of six cysteines (two $\alpha 104$, two $\beta 93$, and two $\beta 112$ ), under the native conditions only two $\beta$ cysteines (one in each $\beta$ ) are the primary residues exposed on the surface. Increasing the molar ratio of PMB to $\mathrm{hHb}$ showed the same pattern of PMB labeling under the native conditions and support that only one cysteine in the $\beta$ unit is accessible by PMB (data not shown). The measured number of surface-exposed cysteines is also consistent with spectrometric measurement requiring multiple titrations (Supplementary Fig. S1(A), see Supporting Information). The results are also consistent with the accessible area of individual cysteines using the theoretical calculation software GETAREA, ${ }^{35}$ by which an increasing order of surface area was found as $\alpha 104, \beta 112$, and $\beta 93$.

\section{Identification of the surface-exposed cysteinyl sites using peptide mapping}

The specific sites of cysteines bound to PMB were further identified by using mass spectrometry peptide mapping. The unlabeled and PMB-labeled proteins were digested using TPCK-modified trypsin. The resultant peptide mixtures were then purified and enriched using C18 Ziptips, and further analyzed using nanoESI-MS. It was expected that 20 peptides (with expected MW >500 Da) could be generated from the trypsin digestion of $\mathrm{hHb}$, as shown in Fig. 2(A). Among these 20 expected peptides, three peptides contain cysteine. In the absence of PMB labeling, only 16 peptides were observed and none of the three cysteine-containing peptides $(\alpha \mathrm{T} 8, \beta \mathrm{T} 7$, and $\beta$ T9) was detectable (bottom trace in Fig. 2(B)). The coverage of the amino acid sequence was $66 \%$. Failure to detect these cysteine-containing peptides is consistent with previous work, ${ }^{36}$ and may be caused by the oxidation and adsorption of cysteines. ${ }^{36,37}$ After PMB labeling of cysteines, the detection sensitivity of the cysteine-containing peptides is greatly enhanced, probably by preventing oxidation and adsorption. As shown in the top trace of Fig. 2(B), all the three PMB-labeled peptides were observed after the denatured $\mathrm{hHb}$ was labeled with PMB. The coverage of the amino acid sequence was enhanced to $86 \%$. Under the nondenaturing conditions of PMB labeling, only one cysteine-containing peptide ( $\beta$ T7) was labeled with PMB (middle trace in Fig. 2(B)). This is consistent with the above result showing 
(A)

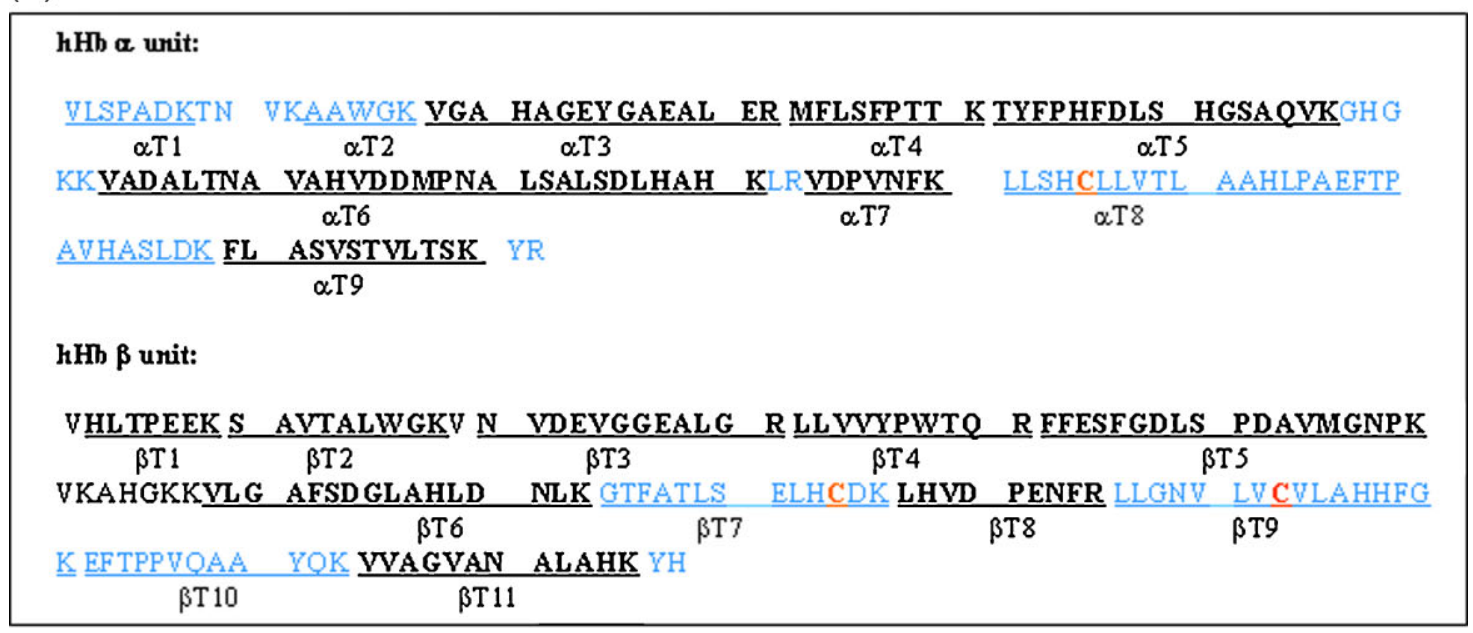

(B)

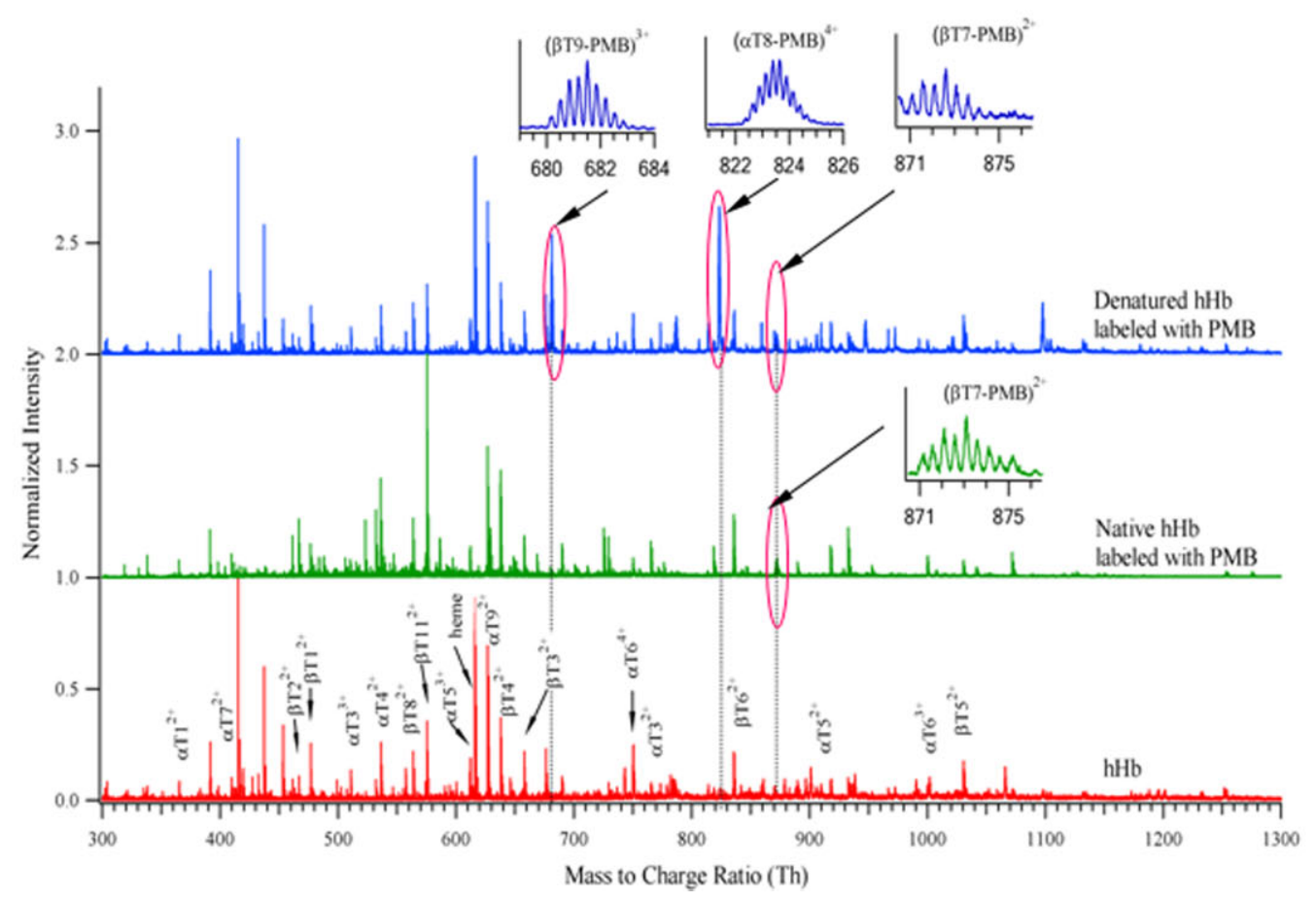

Figure 2. Mapping of PMB-labeled native $\mathrm{hHb}$ and denatured $\mathrm{hHb}$ by trypsin digestion followed by nanoESIMS analysis. (A) Sequence map of $\alpha$ and $\beta$ units of $\mathrm{hHb}$. Note: the sequences written in bold indicate the observed peptides. (B) Full MS spectra for the peptide mixtures obtained from trypsin digestion of unlabeled $\mathrm{hHb}$, labeled native $\mathrm{hHb}$, and labeled denatured $\mathrm{hHb}$ showing the identified peptides. The insets show the unique cluster patterns of PMB-labeled peptides. The presence of PMB-labeled $\beta T 7$ and the absence of PMB-labeled $\beta$ T9 and $\alpha$ T8 from PMB-labeled native hHb suggest that only the cysteine in $\beta$ T7 (Cys93 $\beta$ ) is the exposed cysteine residue in $\mathrm{hHb}$.

that only a single cysteine in the $\beta$ unit was accessible by PMB.

Another attractive feature of PMB labeling is that PMBlabeled peptides have a specific isotopic signature. Mercury has six major isotopes ranging from 197.97 Da to 203.97 Da, with the highest intensity at 201.97 Da. When the mercurycontaining PMB is attached to a peptide, such as $\beta T 7$, it would change the isotopic distribution of the labeled peptide by moving the most intensive isotopic peak from the lower
MW range to the higher MW range of the isotopic cluster, and hence changing the overall pattern of the isotopic clusters. Using the proteomics software MS-Isotope, ${ }^{38}$ we calculated the theoretical isotopic patterns of peptide $\beta T 7$ (from $\mathrm{hHb}$ ) with and without labeling by PMB. There are clear differences in isotopic patterns between labeled and unlabeled peptides. The relative intensity of isotopic peaks agreed well between the theoretical calculation and the experimentally measured values for PMB-labeled $\beta T 7$ 
(Supplementary Fig. S2, see Supporting Information). Thus, such a specific signature could facilitate rapid identification of PMB-labeled peptides from the large pool of unlabeled peptides.

By scanning the whole spectrum to search for such characteristic isotopic clusters, three MS peaks associated with cysteine-containing peptides were identified (Fig. 2(B)), corresponding to $\alpha \mathrm{T} 8, \beta \mathrm{T} 7$, and $\beta \mathrm{T} 9$, respectively. For denatured $\mathrm{hHb}$ with $\mathrm{PMB}$ labeling, three PMB-labeled peptides were all observed (top trace and its insets in Fig. 2(B)). One isotopic cluster with $\mathrm{m} / \mathrm{z}$ ranging from 871 to 874 (highest intensity at $m / z$ of 872.6 ) is a doubly charged species, which corresponds to PMB-labeled peptide $\beta$ T7 containing Cys93 $\beta\left({ }^{83} \mathrm{GTFATLSELHCDK}^{95}\right)$. Another triply charged isotopic cluster in the range of $m / z 680$ to 683 (highest intensity at $m / z$ of 681.5$)$ corresponds to the PMB-labeled peptide $\beta$ T9 $\left({ }^{105}\right.$ LLGNVLVCVLAHHFGK $\left.^{120}\right)$ containing Cys112 $\beta$. The third cluster with $\mathrm{m} / \mathrm{z}$ ranging from 822 to 825 carries four positive charges (highest intensity at $\mathrm{m} / \mathrm{z}$ of 823.4) and corresponds to the PMB-labeled peptide $\alpha \mathrm{T} 8$ containing Cys104 $\alpha$. However, for the native $\mathrm{hHb}$ labeled with PMB (middle trace in Fig. 2(B)), only one doubly charged cluster with $m / z$ ranging from 871 to 874 is observed, which corresponds to $\beta \mathrm{T} 7$ containing Cys93 $\beta$. There is no other isotopic cluster corresponding to PMB-labeled $\beta$ T9 or $\alpha \mathrm{T} 8$. These results indicate that in the native state of $\mathrm{hHb}$, only the cysteine at $\beta 93$ is exposed. The other cysteines at $\beta 112$ and $\alpha 104$ can only be accessible by PMB in the presence of a denaturant such as urea. These results are consistent with the previous report that $\beta 93$ is on the surface of the $\mathrm{hHb}$ tetramer. $^{16}$

\section{Confirmation of PMB labeling at cysteines by peptide sequencing using MS/MS}

For the native $\mathrm{hHb}$ labeled with $\mathrm{PMB}$, the peak with $\mathrm{m} / \mathrm{z}$ of 873 (unit resolution) was selected as the precursor ion and subjected to collision-induced dissociation and time-of-flight (TOF) MS analysis. A series of ions generated during fragmentation of the precursor ions were then compared with the $m / z$ values generated from the theoretical prediction. ${ }^{38}$ The sequence ion of $\mathrm{y}_{2}$ was observed clearly, as shown in Fig. 3. The signal for $y_{3}$ to $y_{10}$ was not clear. Instead, the $m / z$ values of $\mathrm{y}_{3}{ }^{*}$ to $\mathrm{y}_{10}{ }^{*}$ are all $321 \mathrm{Th}$ higher than the theoretical values for the respective $\mathrm{y}_{3}$ to $\mathrm{y}_{10}$ ions. There is no peak with $m / z 321$ Th higher than those for $\mathrm{y}_{1}$ or $\mathrm{y}_{2}$. These results indicate that one PMB molecule is bound to Cys93 $\beta$ in the peptide of $\beta$ T7.

\section{Application of the method in identification of exposed cysteines in $\mathrm{rHb}$}

With the identification of exposed cysteines in human hemoglobin, we further applied the method to characterize the exposed cysteines in rat hemoglobin $(\mathrm{rHb})$. $\mathrm{rHb}$ contains 10 free cysteine residues, with three in each $\alpha$ unit and two in each $\beta$ unit. First, we examined the number of exposed cysteines by using excess $\mathrm{PMB}$ to react with $\mathrm{rHb}$ followed by nanoESI-MS analysis of the mixture. As shown in the MS spectrum (bottom trace of Fig. 4), wild-type $\mathrm{rHb}\left(\mathrm{rHb}^{\mathrm{WT}}\right)$

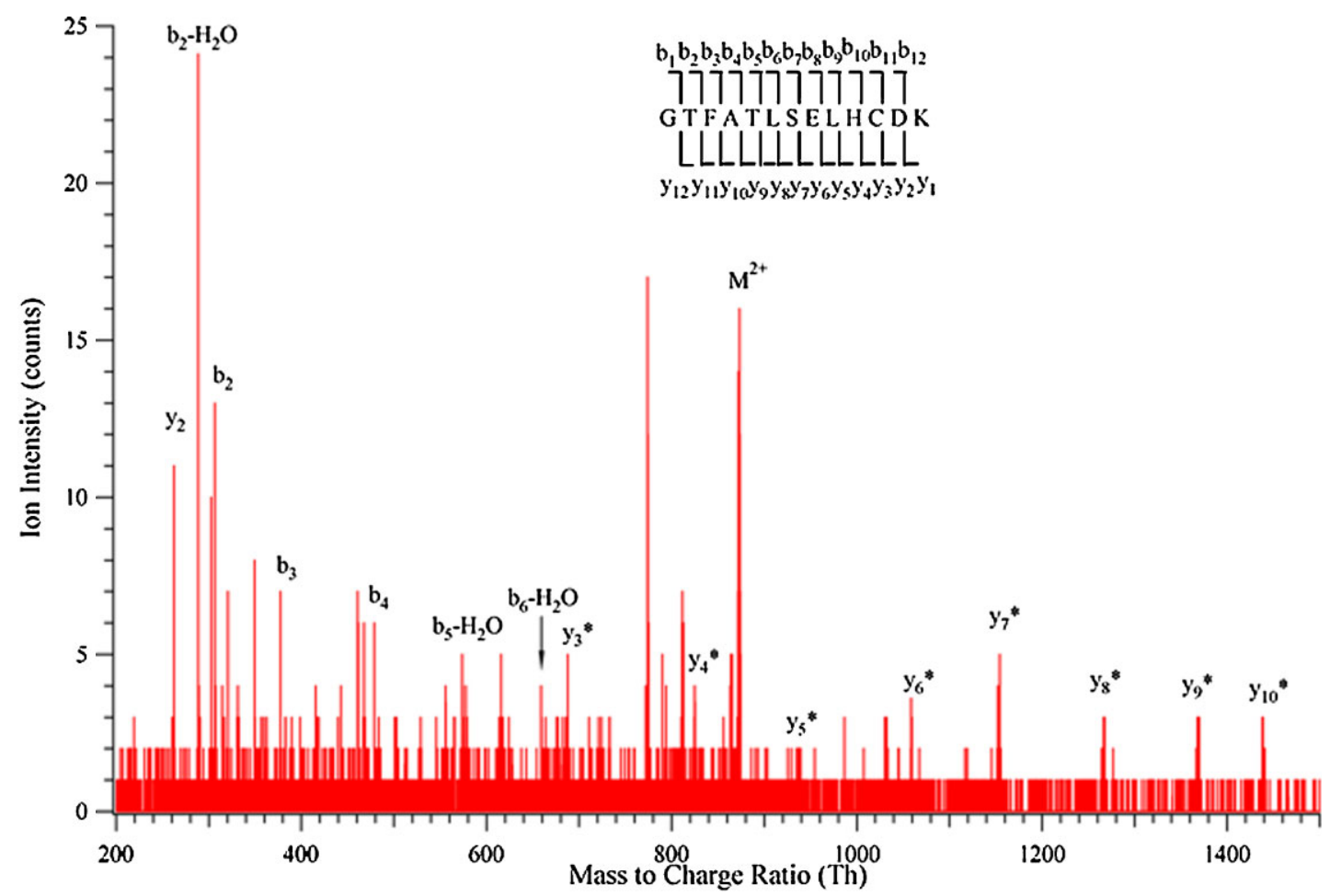

Figure 3. MS/MS analysis of $\beta T 7^{2+}$ of $\mathrm{hHb}$ demonstrating that $\mathrm{PMB}$ is labeled at the third position from the C-terminal of the $\beta T 7$ fragment and indicating that Cys93 $\beta$ is the exposed cysteine in $\mathrm{hHb}$. The precursor ions $\left(\beta T 7^{2+}\right)$ were selected in the second quadrupole with $\mathrm{m} / \mathrm{z}$ of $873 \mathrm{Th}$, fragmented by collision-induced dissociation before MS analysis. Mainly, a series of y ions are observed, but with an $\mathrm{m} / \mathrm{z}$ increment of 321 Th for the $y_{3}$ to $y_{10}$ ions, indicating that PMB is labeled at the $y_{3}$ position, which is Cys93ß. 


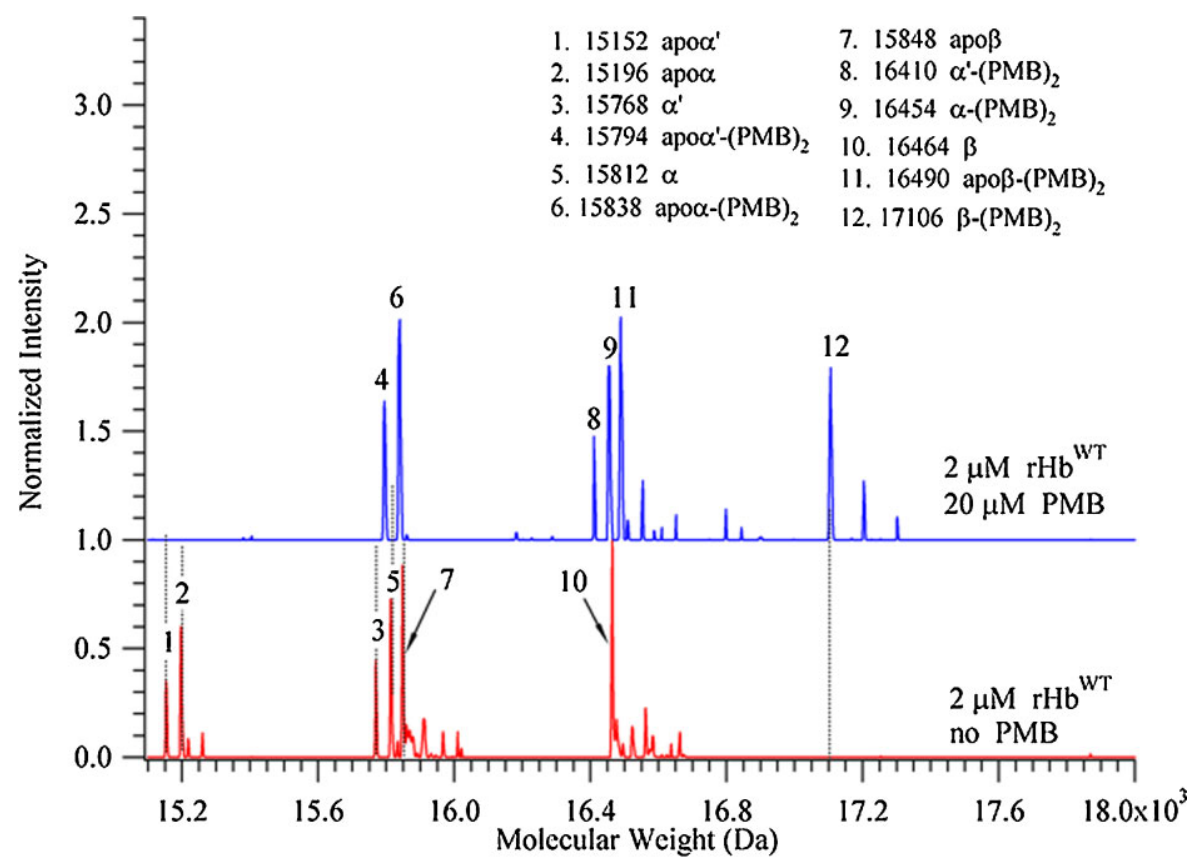

Figure 4. The deconvoluted mass spectra obtained from nanoESI-MS analysis of the unlabeled and $\mathrm{PMB}$-labeled native $\mathrm{rHb}$ (wild-type) showing the formation of the complexes of subunits of native $\mathrm{rHb}$ and $\mathrm{PMB}$ with a binding stoichiometry of $1: 2$ and indicating the presence of eight exposed cysteines in the $\mathrm{rHb}$ tetramer. Major peaks were identified with molecular mass and listed in the figure.

displays six major peaks corresponding to apo $\alpha^{\prime}$ (peak 1, $15152 \mathrm{Da}$, a variant of the $\alpha$ unit), apo $\alpha$ (peak 2, $15196 \mathrm{Da}$ ), intact $\alpha^{\prime}$ (peak 3, $15768 \mathrm{Da}$ ), intact $\alpha$ (peak 5, $\left.15812 \mathrm{Da}\right)$, apo $\beta$ (peak 7, $15848 \mathrm{Da}$ ), and intact $\beta$ (peak 10, $16464 \mathrm{Da}$ ). After reaction with a 10 -fold molar excess of $\mathrm{PMB}$, all these major peaks are shifted to higher molecular mass positions, all with a mass increment of 642 Da (peaks 4, 6, 8, 9, 11, and 12 in top trace of Fig. 4). These results indicate the incorporation of two PMB molecules, suggesting the existence of two accessible cysteines exposed in each unit. Although three cysteines $(\alpha 13, \alpha 104$, and $\alpha 111)$ are present in each $\alpha$ unit of $\mathrm{rHb}^{\mathrm{WT}}$, no peak corresponding to the complex of one apo/intact $\alpha$ unit bound with three PMB molecules is observed, indicating that one cysteine is shielded by the protein. The variant of the $\alpha$ unit of $\mathrm{rHb}^{\mathrm{WT}}$, the $\alpha^{\prime}$ unit, which has the same three cysteines, displays the same mass shift pattern. Since the $\beta$ unit of $\mathrm{rHb}^{\mathrm{WT}}$ contains only two cysteine residues ( $\beta 93$ and $\beta 125)$, incorporation of two PMB molecules into one $\beta$ unit indicates that both cysteines are reactive with $\mathrm{PMB}$.

With use of a much higher molar ratio of PMB titration for $\mathrm{rHb}^{\mathrm{WT}}$ (24:1), the nanoESI-MS analysis consistently suggests the presence of two surface-exposed cysteines for each observed unit of $\mathrm{rHb}$ (including the $\alpha$ unit, the $\alpha^{\prime}$ unit and the $\beta$ unit) (data not shown). Combining the information obtained from both the $\alpha$ and $\beta$ units of $\mathrm{rHb}^{\mathrm{WT}}$, it is concluded that there are eight surface-exposed cysteines per $\mathrm{rHb}$ tetramer ( $2 \alpha$ and $2 \beta$ units). These results are similar to those obtained by spectrophotometric titration from which about seven exposed cysteines are suggested (Supplementary Fig. S1(B), see Supporting Information).

We further examined the position of the exposed cysteines in $\mathrm{rHb}$. For identification of exposed cysteinyl sites in $\mathrm{rHb}$, we used a hemoglobin extracted from frozen red blood cells of an F344 rat, labeled with excess PMB under native conditions, and analyzed the PMB-labeled sites. Theoretically, it is expected that 22 peptides (with expected MW $>500$ $\mathrm{Da}$ ) could be generated from trypsin digestion of $\mathrm{rHb}$, as shown in Fig. 5(A). Among these 22 expected peptides, there are four cysteine-containing peptides. Without PMB labeling, there are 16 peptides observed. Similar to $\mathrm{hHb}$, none of the cysteine-containing peptides is detectable (bottom trace in Fig. 5(B)). The coverage of amino acid sequence is $65 \%$. In comparison, up to 19 peptides including three cysteinecontaining peptides ( $\alpha \mathrm{T} 9, \beta \mathrm{T} 8$, and $\beta \mathrm{T} 11)$ were observed for PMB-labeled native $\mathrm{rHb}^{\mathrm{F} 344}$ (Fig. 5(B) and its inset). In this case, the coverage of the amino acid sequence approached over $84 \%$. The peaks with $m / z$ at $926.62\left(4^{+}\right), 741.53\left(5^{+}\right)$, and $618.13\left(6^{+}\right)$ are due to the complex of two PMB molecules with the peptide $\alpha$ T9 which contains Cys $104 \alpha$ and Cys $111 \alpha\left({ }^{100}\right.$ FLSHCLLVTL ACHHPGDFTPAMHASLDK ${ }^{127}$ ), indicating that both Cys104 $\alpha$ and Cys $111 \alpha$ are surface-exposed. In contrast, no doubly charged cysteine-containing $\alpha \mathrm{T} 2$ peptide with a PMB label was detected in the $m / z$ range of 464-465 even when $\mathrm{rHb}$ was reacted with excess PMB (inset of Fig. 5(B)). The result indicates that the cysteine at $\alpha 13$ belonging to the $\alpha \mathrm{T} 2$ fragment is not surface-exposed. This is consistent with the simulation of the three-dimensional structure of $\mathrm{rHb}$, in which cysteine at $\alpha 13$ was found to locate in a hydrophobic milieu. ${ }^{32}$

Similarly, the observed peptide ions with $\mathrm{m} / \mathrm{z}$ of $889.76\left(2^{+}\right)$ and $593.51\left(3^{+}\right)$correspond to the complex of one PMB and one $\beta$ T8 $\left({ }^{83}\right.$ GTFAHLSELHCDK $\left.{ }^{95}\right)$ containing Cys93 $\beta$, and the peptide ions with $m / z$ of $831.79\left(2^{+}\right)$are due to the complex of one PMB and one $\beta$ T11 $\left({ }^{121}\right.$ EFTPCA$\mathrm{QAAFQK}^{132}$ ) containing Cys $125 \beta$. These results indicate that 
(A)

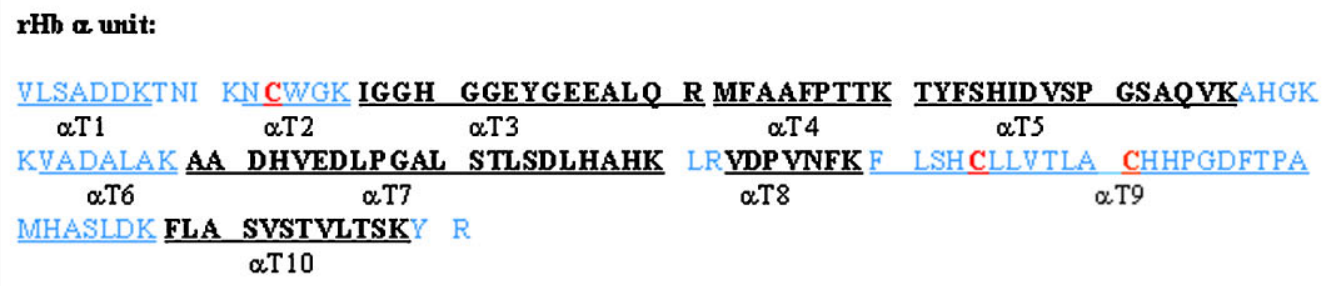

(B)

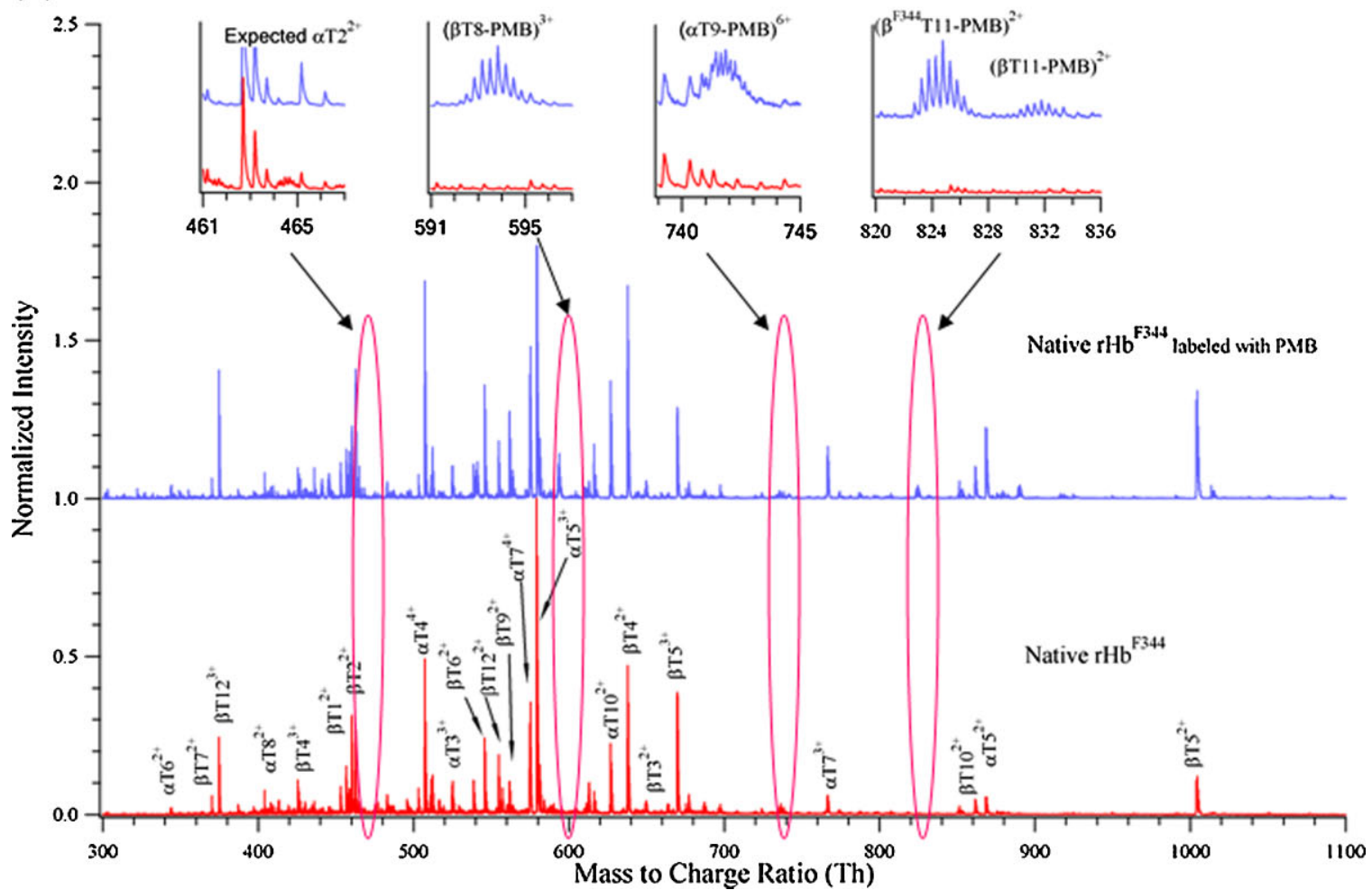

Figure 5. NanoESI-MS analysis of the trypsin digest from $\mathrm{rHb}^{\mathrm{F} 344}$ and its $\mathrm{PMB}$-labeled products. (A) Sequence map of $\alpha$ and $\beta$ units of $\mathrm{rHb}^{\mathrm{F} 344}$. The sections written in bold annotate the observed peptides. (B) Full MS spectra for the peptide mixtures obtained from trypsin digestion of both unlabeled and labeled native $\mathrm{rHb}^{\mathrm{F} 344}$ showing the identified peptides. The insets show the unique cluster patterns of PMB-labeled peptides. The presence of ions related to $\alpha \mathrm{T} 9(\mathrm{PMB})_{2}$ from $\alpha 100$ to $\alpha 127, \beta \mathrm{T} 8(\mathrm{PMB})$ from $\beta 83$ to $\beta 95$, and $\beta \mathrm{T} 11$ (PMB) from $\beta 121$ to $\beta 132$ indicates that two cysteines in the $\alpha$ unit $(\alpha 104$ and $\alpha 111)$ and two cysteines in the $\beta$ unit ( $\beta 93$ and $\beta 125)$ were labeled by PMB, providing evidence for surface exposure of these cysteines.

both cysteines at $\beta 93$ and $\beta 125$ of $\mathrm{rHb}$ are surface-exposed, which is consistent with the result obtained by single PMB titration followed by direct nanoESI-MS analysis in which two PMB molecules can bind to one $\beta$ unit of $\mathrm{rHb}$ (Fig. 4).

It is surprising that one PMB-labeled isotopic cluster (peak with highest intensity at $m / z$ of $824.76, z=2$ ) was observed with an intensity about 5-fold higher but a molecular weight of 14 Da less than that of doubly charged PMB-labeled $\beta$ T11. It does not match with any expected cysteine-containing peptides, which are the only residues labeled specifically by high affinity PMB. Recalling that the major $\beta$ unit of $\mathrm{rHb}$ from the F344 rat was $14 \mathrm{Da}$ smaller than the wild-type $\mathrm{rHb}, 32,39$ we hypothesize that the 14 Da loss in MW is due to mutation 
of a single amino acid residue within the range of 121-132 $(\beta \mathrm{T} 11)$ in the $\beta$ unit of $\mathrm{rHb}^{\mathrm{F} 344}$. By searching for mutations of wild-type $\mathrm{rHb}$ in the Swiss-Prot database, it was found that there were no mutations reported on cysteines at any of the five positions of $\mathrm{rHb}^{\mathrm{WT}}(\alpha 13, \alpha 104, \alpha 111, \beta 93$ and $\beta 125)$, or on other residues which would be mutated to generate new cysteines. However, there was one mutation on $\beta 123$ where ${ }^{123} \mathrm{~T}$ (threonine) can be mutated to ${ }^{123} \mathrm{~S}$ (serine), which could result in a lowering of the MW by 14 Da. Among eight mutations reported on both $\alpha$ and $\beta$ units of $\mathrm{rHb}$ in the SwissProt database, only this mutation matches the observed peak $(m / z$ at $824.76, z=2)$. Therefore, the peak at $m / z$ of $824.76\left(2^{+}\right)$ was assigned as one PMB-labeled peptide $\beta^{\mathrm{F} 344} \mathrm{~T} 11\left({ }^{121} \mathrm{EFSP}-\right.$ $\mathrm{CAQAAFQK}^{132}$ ). These results also suggest that the mutation of $\beta 123 \mathrm{~T} \rightarrow \beta 123 \mathrm{~S}$ in $\mathrm{rHb}$ may not significantly change the exposure of the neighboring cysteine ( $\beta 125)$.

Taken together, the eight cysteines in one tetramer of $\mathrm{rHb}$ (two $\alpha 104$, two $\alpha 111$, two $\beta 93$, and two $\beta 125$ ) are surfaceexposed, and only the two cysteines at $\alpha 13$ are shielded in a hydrophobic pocket.

\section{Evaluation of the relative extent of accessibility to the surface cysteines}

We also made use of PMB labeling followed by the MS analytical technique to evaluate the relative accessibility of the suggested surface-exposed cysteines, thereby providing information about subtle differences between surfaceexposed cysteines. This was achieved by PMB titration of $\mathrm{rHb}$ combined with nanoESI-MS analysis. In this case, $\mathrm{rHb}^{\mathrm{F} 344}$ was used as an example. By assuming the sum of each unit and their PMB complexes as $100 \%$, the relative intensity of each species was estimated from the deconvoluted mass spectra in Supplementary Fig. S3 (see Supporting Information), and plotted against the concentration of PMB. When $\mathrm{rHb}(2.0 \mu \mathrm{M})$ was titrated with $2.0 \mu \mathrm{M} \mathrm{PMB}$, about $79 \%$ of the $\beta$ unit was labeled by one or two PMB molecules (Fig. 6(B)). Meanwhile, only $13 \%$ of the $\alpha$ unit was labeled by one PMB molecule and no $\alpha$ unit was labeled by two PMB molecules (Fig. 6(A)). When the same amount of $\mathrm{rHb}$ was titrated with $4.0 \mu \mathrm{M} \mathrm{PMB}$, about $85 \%$ of the $\beta$ unit was labeled by $\mathrm{PMB}$, of which $55 \%$ was labeled by two PMB molecules. In contrast, only about $32 \%$ of the $\alpha$ unit was labeled by $\mathrm{PMB}$, and mostly by one PMB. These results suggest that the two surface-exposed cysteines (Cys93 $\beta$ and Cys125 $\beta$ ) in the $\beta$ unit are much more reactive to PMB than those in the $\alpha$ unit (Cys104 $\alpha$ and Cys111 $\alpha$ ), indicating a much higher degree of exposure for the cysteines in the $\beta$ unit. These results are also consistent with the theoretical calculation of GETAREA, ${ }^{35}$ by which we found that the surface areas for $\beta 93$ and $\beta 125$ are much greater than those for cysteines in the $\alpha$ unit.

We have identified previously two highly reactive cysteines in $\mathrm{rHb}, \mathrm{Cys} 13 \alpha$ and Cys $125 \beta$. Cys $13 \alpha$ is a highly reactive site for binding with dimethylarsinous acid $\left(\mathrm{DMA}^{\mathrm{III}}\right){ }^{32,33}$ a key metabolite formed in human exposure to naturally occurring arsenic in the environment. ${ }^{40,41}$ Cys $125 \beta$ reacts specifically with an endogenous anti-oxidant, glutathione. ${ }^{33}$ The study reported here suggests that the DMA $^{\mathrm{III}}$-binding site, Cys $13 \alpha$, is not exposed on the surface. Its high reactivity toward $\mathrm{DMA}^{\mathrm{III}}$ is probably due to a
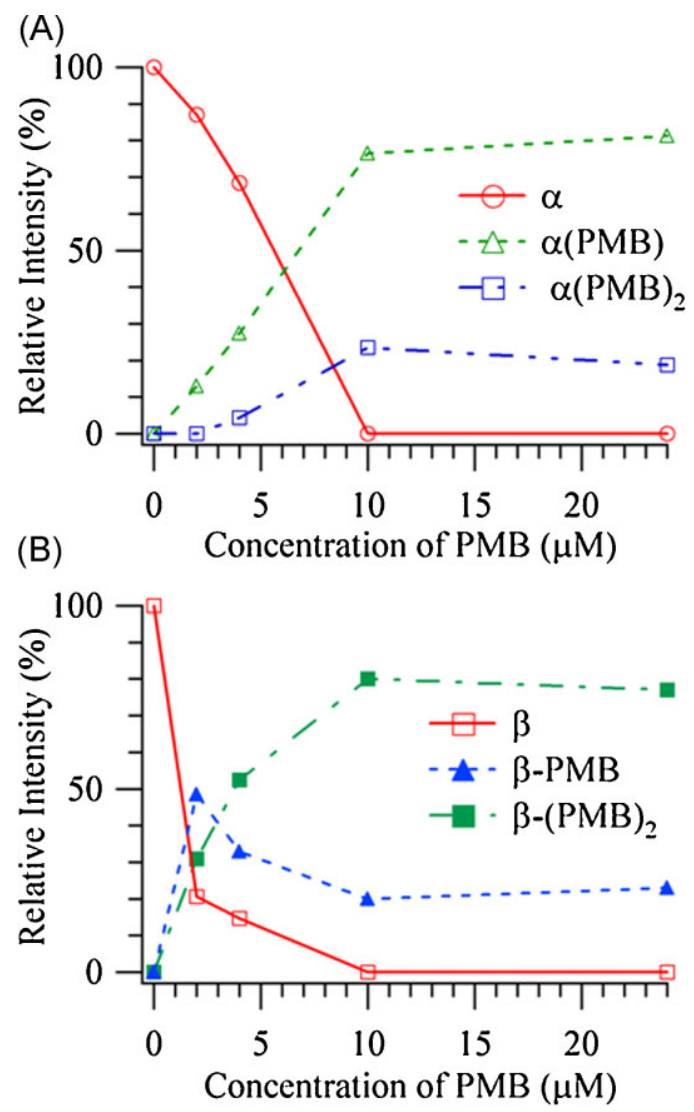

Figure 6. The relative intensity of the $\alpha$ and $\beta$ units of $\mathrm{rHb}$ and their $1: 1$ and $1: 2$ complexes with PMB versus the titrated concentrations of PMB. The intensities were read out from Supplementary Fig. S3 (see Supporting Information).

moderately hydrophobic pocket that is suitable for entry of DMA $^{\mathrm{III}}$. A preliminary molecular dynamic simulation supports this notion. ${ }^{32}$ On the other hand, the surfaceexposed Cys $125 \beta$ readily reacts with larger and more hydrophilic glutathione.

Previously, Gao and Wang ${ }^{42}$ applied methylglyoxal together with a MS/MS technique to examine the surfaceexposed arginine residues in human hemoglobin. Here we combine the high sensitivity and resolution of nanoESI Q-TOF MS with the high selectivity of a monovalent mercury compound for evaluation of solvent-exposed cysteines in both hemoglobin of human and rat. We demonstrate here two advantages of PMB labeling. One is the ability to rapidly determine the number of surface-exposed cysteines by a single titration. When a protein is labeled by excess PMB, the number of surface-exposed cysteines can be derived from the number of complexed PMB per protein molecule, which can be directly read out from the deconvoluted mass spectrum because its large mass shift (321 Da per complexed PMB molecule). The other is that PMB labeling introduces a unique isotopic fingerprint to the peptides containing surfaceexposed cysteine(s) in peptide mapping, which facilitates fast identification of the exposed cysteinyl sites in proteins.

PMB labeling is highly selective to the free and exposed cysteines. The high affinity is primarily derived from the electrophilic property of the mercury atom and the nucleophilic characteristics of the sulfhydryl group or its ionized form - the thiolate anion. ${ }^{23,29,43}$ The dissociation 
constants for the mercaptide formed between cysteine and mercuric ion or methyl mercury are below $\mathrm{fM}^{29}$ The high affinity of PMB for sulfhydryl groups, together with its bulky size and hydrophilic nature, allows it to selectively bind to the cysteines on the protein surface with good stability.

In addition to the high affinity of PMB with surfaceexposed cysteines, our work hints that PMB labeling can retain the conjugation information on reactive surfaceexposed cysteines. It was observed that the glutathioneconjugated $\beta$ unit (peak 11, MW 16756 Da) could be formed in $\mathrm{rHb}^{\mathrm{F} 344}$ extracted from rat red blood cells (Supplementary Fig. S3, see Supporting Information). With titration using excess PMB, no significant displacement of conjugated glutathione from the glutathione-conjugated $\beta$ unit by PMB was observed. On the contrary, a complex of the $\beta$ unit that contains one PMB and one conjugated gluthathione (peak 13, 17077 Da) was observed. Previous work has suggested that glutathione predominantly binds to the highly reactive Cys $125 \beta$ in $\mathrm{rHb}^{\mathrm{F} 344}$, 33 which is also a target of high affinity PMB as described in this work. These results suggest that high affinity $\mathrm{PMB}$ cannot displace the conjugated glutathione from surface-exposed Cys125 $\beta$. This may indicate that PMB titration probably has the capacity to retain the information on the conjugation state of surfaceexposed cysteines, which is useful for the study of the cysteine redox status of various proteins that can be regulated by endogenous oxidative stress.

Cysteine is one of the highly conservative amino acids in most proteins, and often plays crucial roles in the structure and function of proteins. This is mainly derived from the redox properties of the sulfhydryl group, the ability to chelate metal ions, and to stabilize proteins by forming disulfide bonds. ${ }^{23,44}$ The cysteines on the protein surface are often the target of the endogenous/exogenous substrates such as glutathione, $\mathrm{NO}$, and other reactive metabolites of drugs/toxins, etc. ${ }^{12-14,44,45}$ Modification by these substrates can lead to a range of cellular responses. We provide here a convenient approach for fast screening and identification of biochemically significant cysteines on proteins, exploring their associated conformational changes caused by various stresses, post-translational modifications, enzyme-substrate reactions, protein-protein interactions, and protein assembly, which will facilitate the detailed down-stream functional studies of these proteins.

\section{SUPPORTING INFORMATION}

Additional supporting information may be found in the online version of this article.

\section{Acknowledgements}

This work was supported by grants from the National Natural Science Foundation of China (Nos. 20737003, 20677066, and 20621703) and the National Basic Research Program of China (973 program, No. 2007CB407305) to Dr. H. Wang, and Alberta Heritage Foundation for Medical Research Post-doctoral Fellowship to Dr. M. Lu. The authors also thank Dr. Samuel M. Cohen and Ms. Lora L. Arnold of the University of Nebraska Medical Center for the blood samples from F344 rats.

\section{REFERENCES}

1. Gupta N, Ragsdale SW. J. Biol. Chem. 2008; 283: 28721.

2. Zhang DD, Hannink M. Mol. Cell. Biol. 2003; 23: 8137.

3. Giese NA, Robbins KC, Aaronson SA. Science 1987; 236: 1315.

4. Guo R, Rigolet P, Zargarian L, Fermandjian S, Xi XG. Nucleic Acids Res. 2005; 33: 3109.

5. Murakami T, Nojiri M, Nakayama H, Odaka M, Yohda M, Dohmae N, Takio K, Nagamune T. Protein. Sci. 2000; 9: 1024.

6. Mortusewics O, Ame JC, Schreiber V, Leonhardt H. Nucleic Acids Res. 2007; 35: 7665.

7. Zhong L, Arner ESJ, Holmgren A. Proc. Natl. Acad. Sci. USA 2000; 97: 5854.

8. Fratelli M, Demol H, Puype M, Casagrande S, Eberini I, Salmona M, Bonetto V, Mengozzi M, Duffieux F, Miclet E, Bachi A, Vandekerckhove J, Gianazza E, Ghezzi P. Proc. Natl. Acad. Sci. USA 2002; 99: 3505.

9. Mannick JB. Amino Acids 2007; 32: 523.

10. Kumar A, Wu H, Collier-Hyams LS, Hansen JM, Li T, Yamoah K, Pan ZQ, Jones DP, Neish AS. EMBO J. 2007; 26: 4457.

11. Wakabayashi N, Dinkova-Kostova AT, Holtzclaw WD, Kang M, Kobayashi A, Yamamoto M, Kensler TW, Talalay P. Proc. Natl. Acad. Sci. USA 2004; 101: 2040.

12. Rainwater R, Pars D, Anderson ME, Tegtmeyer P, Mann K. Mol. Cell. Biol. 1995; 15: 3892.

13. Velu CS, Niture SK, Doneanu CE, Pattabiraman N, Srivenugopal KS. Biochemistry 2007; 46: 7765.

14. Martinez-Ruiz A, Villanueva L, de Orduna CG, Lopez Ferrer D, Higueras MA, Tarin C, Rodriguez-Crespo I, Vazquez J, Lamas S. Proc. Natl. Acad. Sci. USA 2005; 102: 8525

15. Casagrande $S$, Bonetto V, Fratelli M, Gianazza E, Eberini I, Massignan T, Salmona M, Chang G, Holmgren A, Ghezzi P. Proc. Natl. Acad. Sci. USA 2002; 99: 9745.

16. Rieder RF, Oski FA, Clegg JB. J. Clin. Invest. 1969; 48: 1627.

17. Johnson CP, Tang H-Y, Carag C, Speicher DW, Discher DE. Science 2007; 317: 663.

18. Ha JH, Loh SN. Nat. Struct. Biol. 1998; 5: 730.

19. Silverman JA, Harbury PB. J. Biol. Chem. 2002; 277: 30968.

20. Olson J. J. Biol. Chem. 1976; 251: 441.

21. Jager M, Michalet X, Weiss S. Protein Sci. 2005; 14: 2059

22. Tinguely JN, Wermuth B. FEBS J. 1999; 260: 9.

23. Britto PJ, Knipling L, Wolff J. J. Biol. Chem. 2002; 277: 29018.

24. Belghazi M, Klett D, Cahoreau C, Combarnous Y. Mol. Cell. Endocrinol. 2006; 247: 175.

25. Griffiths SW, King J, Cooney C. J. Biol. Chem. 2002; 277: 25486.

26. Dunham TD, Farrens DL. J. Biol. Chem. 1999; 274: 1683.

27. Riordan JF, Valee BL. Methods Enzymol. 1972; 25: 449.

28. Guidotti G, Konigsburg W. J. Biol. Chem. 1964; 239: 1474.

29. Simpson RB. J. Am. Chem. Soc. 1961; 83: 4711.

30. Zaluzec EJ, Gage DA, Watson JT. J. Am. Soc. Mass Spectrom. 1994; 5: 359.

31. Raftery FJ. Anal. Chem. 2008; 80: 3334.

32. Lu M, Wang H, Li X-F, Lu X, Arnold LL, Cohen SM, Le XC. Chem. Res. Toxicol. 2007; 20: 27.

33. Lu M, Wang H, Wang Z, Li X-F, Le XC. J. Proteome Res. 2008; 7: 3080 .

34. http://www.expasy.com

35. http://curie.utmb.edu/getarea.html.

36. Sechi S, Chait BT. Anal. Chem. 1998; 70: 5150.

37. Atsriku C, Scott GK, Benz CC, Baldwin MA. J. Am. Soc. Mass Spectrom. 2005; 16: 2017.

38. http:// prospector.ucsf.edu/cgi-bin.

39. Lu M, Wang H, Li X-F, Lu X, Cullen WR, Arnold LL, Cohen SM, Le XC. Chem. Res. Toxicol. 2004; 17: 1733.

40. Le XC, Lu X, Ma M, Cullen WR. Anal. Chem. 2000; 72 5172.

41. NRC. Arsenic in Drinking Water. National Research Council, National Academy Press: Washington, DC, 1999.

42. Gao Y, Wang YS. Biochemistry 2006; 45: 15654.

43. Brewer CF, Riehm JP. Anal. Biochem. 1967; 18: 248.

44. Ferranti P, Sannolo N, Mamone G, Fiumer I, Carbone V, Tornqvist M, Bergman A, Malorni A. Carcinogenesis 1996; 17: 2661.

45. Tummala M, Ryzhov V, Ravi K, Black SM. DNA Cell Biol. 2008; 27: 25 . 